Acta Crystallographica Section F

Structural Biology

and Crystallization

Communications

ISSN 1744-3091

Poorva D. Dharkar, P. Anuradha, Sushama M. Gaikwad and

\section{G. Suresh*}

Division of Biochemical Sciences, National Chemical Laboratory, Pune 411008, India

Correspondence e-mail: cg.suresh@ncl.res.in

Received 16 December 2005

Accepted 23 January 2006

\section{Crystallization and preliminary characterization of a highly thermostable lectin from Trichosanthes dioica and comparison with other Trichosanthes lectins}

A lectin from Trichosanthes dioica seeds has been purified and crystallized using 25\% $(w / v)$ PEG 2K MME, 0.2 $M$ ammonium acetate, $0.1 M$ Tris- $\mathrm{HCl}$ pH 8.5 and $50 \mu \mathrm{l} 0.5 \%(w / v) n$-octyl $\beta$-D-glucopyranoside as thick needles belonging to hexagonal space group $P 6_{4}$. Unit-cell parameters were $a=b=167.54, c=77.42 \AA$. The crystals diffracted to a Bragg spacing of $2.8 \AA$. Both the structures of abrin-a and T. kirilowii lectin could be used as a model in structure determination using the molecular-replacement method; however, T. kirilowii lectin coordinates gave better values of reliability and correlation parameters. The thermal, chemical and $\mathrm{pH}$ stability of this lectin have also been studied. When heated, its haemagglutination activity remained unaffected up to $363 \mathrm{~K}$. Other stability studies show that $4 M$ guanidinium hydrochloride $(\mathrm{Gdn}-\mathrm{HCl})$ initiates unfolding and that the protein is completely unfolded at $6 \mathrm{M} \mathrm{Gdn-HCl}$. Treatment with urea resulted in a total loss of activity at higher concentrations of denaturant with no major structural changes. The protein remained stable over a wide $\mathrm{pH}$ range, from $\mathrm{pH} 6$ to $\mathrm{pH} 12$, except for partial unfolding at extremely alkaline $\mathrm{pH}$. The role of disulfide bonds in the protein stability was found to be insignificant. Rayleigh light-scattering studies showed no molecular aggregation in any of the extreme treated conditions. The unusual stability of this lectin resembles that of type II ribosome-inactivating proteins (type II RIPs), which is also supported by structure determination. The structural features observed in a preliminary electron-density map were compared with the other two available Trichosanthes lectin structures.

\section{Introduction}

Lectins are carbohydrate-binding proteins that occur ubiquitously in nature and exhibit important biological properties such as bloodgroup specificity, preferential agglutination of tumour cells and mitogenicity (Lis \& Sharon, 1991). In addition, they serve as interesting models for studying the folding and unfolding pathways of oligomeric proteins. The interactions that stabilize the protein structures are electrostatic and hydrophobic interactions, hydrogen bonds and disulfide linkages. Conditions that disturb these stabilizing forces affect the native conformation of the protein, destroying its biological activity. Of the large number of lectins studied, the relationship between structure, conformation and stability has only been established for a few proteins (Ahmed et al., 1998; Reddy et al., 1999; Gaikwad et al., 2002; Gaikwad \& Khan, 2003; Sahasrabuddhe et al., 2004).

From our studies, we have found that the two Trichosanthes lectins, one from $T$. dioica and the other from $T$. anguina, belonging to the Cucurbitaceae family show unusual stability towards denaturation conditions. T. anguina lectin has been independently studied in detail (Komath et al., 1998; Komath \& Swamy, 1999; Anuradha \& Bhide, 1999; Sultan et al., 2004; Sultan \& Swamy, 2005). Both the above lectins are galactose-specific, preferentially binding the $\beta$-anomer of galactose, and are heterodimers of two nonidentical subunits joined by disulfide bonds. T. anguina lectin has a putative histidine residue (Komath et al., 1998) whilst $T$. dioica lectin has a tyrosine at the sugarbinding site (Sultan et al., 2004). Crystallization of the T. anguina lectin has previously been reported and it has been identified as a type II ribosome-binding protein (type II RIP; Manoj et al., 2001). Four other type II RIPs, ricin (Montfort et al., 1987), abrin-a (Tahirov
(C) 2006 International Union of Crystallography All rights reserved 
et al., 1995), mistletoe lectin (Krauspenhaar et al., 1999) and T. kirilowii lectin (Li et al., 2001), have been structurally characterized. Type II RIPs are cytotoxic heterodimeric proteins with a lectin-like $B$ chain mostly specific towards galactose and an $A$ chain showing glycosidase activity (Hartley \& Lord, 2004).

In this paper, we describe the crystallization and preliminary characterization of $T$. dioica lectin, the evidence of its highly thermostable nature and also the effect of chemical denaturants and $\mathrm{pH}$ on its stability. The structure determination using molecular replacement and the gross structural features in comparison with the known structures of $T$. anguina and $T$. kirilowii lectins are highlighted.

\section{Materials and methods}

\subsection{Materials}

Guanidinium hydrochloride ( $\mathrm{Gdn}-\mathrm{HCl}$ ) was obtained from Sigma (USA). The lectin from T. dioica was purified following a procedure described by Anuradha \& Bhide (1999) for T. anguina lectin. Buffers used were glycine- $\mathrm{HCl}$ for the $\mathrm{pH}$ range $2-3$, acetate for $\mathrm{pH} 4$, citrate-phosphate for $\mathrm{pH} 5$, phosphate for $\mathrm{pH} 7$, Tris- $\mathrm{HCl}$ for $\mathrm{pH} 8-9$ and glycine- $\mathrm{NaOH}$ for $\mathrm{pH} 10-12$ (all at $100 \mathrm{~m} M$ concentration). The stock solutions of $8 M \mathrm{Gdn}-\mathrm{HCl}$ and $10 M$ urea were prepared in phosphate buffer $\mathrm{pH} 7$ and filtered through a $0.45 \mu \mathrm{m}$ filter. Crystal Screen solutions were obtained from Hampton Research (USA). The Tris, PEG 2K MME, $n$-octyl $\beta$-D-glucopyranoside and glycerol used for crystallization experiments were obtained from Sigma (USA). Other chemicals used were obtained locally and were of analytical reagent grade.

\subsection{Fluorescence studies}

The effect of temperature on stability was studied by incubating the protein samples $(1.8 \mu M)$ at temperatures in the range $303-368 \mathrm{~K}$ for $15 \mathrm{~min}$ each. Haemagglutination activity was checked simultaneously by removing aliquots of the samples. The protein samples $(1.8 \mu M)$ were equilibrated for $4 \mathrm{~h}$ at the required denaturant (Gdn$\mathrm{HCl}$ or urea) concentration at $303 \mathrm{~K}$. The intrinsic tryptophan fluorescence emission of the protein was monitored in $1 \mathrm{~cm}$ quartz cell in the $300-400 \mathrm{~nm}$ range, when excited at $280 \mathrm{~nm}$, in a PerkinElmer LS 50B spectrofluorimeter with attached circulating water bath. Excitation and emission band passes of $5 \mathrm{~nm}$ each were used. The activity of the sample was measured at the same time. These studies were also carried out with the protein samples in the presence of the reducing agents DTT and $\beta$-mercaptoethanol $(5 \mathrm{mM})$.

\subsection{Determination of the lectin activity}

Twofold serial dilution of the lectin in saline medium was carried out in a microtitre plate. A $3 \%(v / v)$ erythrocyte suspension was prepared by repeated washing of red blood cells (RBCs) with saline until all proteins were removed and diluting the pellet with saline. $100 \mu \mathrm{l}$ of this suspension was added to the serially diluted lectin in saline and incubated for 30-60 min at room temperature. Haemagglutination was recorded after $1 \mathrm{~h}$.

\subsection{Light-scattering studies}

Rayleigh light-scattering experiments were carried out using the spectrofluorimeter to monitor protein aggregation during $\mathrm{Gdn}-\mathrm{HCl}-$ induced and thermal denaturation. Both excitation and emission wavelengths were set at $400 \mathrm{~nm}$ and the time-dependent change in scattering intensity was monitored.

\subsection{Renaturation studies}

$200 \mu \mathrm{l}$ aliquots were drawn from the samples treated with different concentrations of $\mathrm{Gdn}-\mathrm{HCl}$ as described above and diluted ten times with $100 \mathrm{~m} M$ buffer $\mathrm{pH}$ 7.0. The fluorescence spectra of the diluted samples were recorded after $24 \mathrm{~h}$ and the activity was assayed. A protein sample in the absence of $\mathrm{Gdn}-\mathrm{HCl}$, but treated under identical conditions, was used as a control. The protein samples treated at high temperatures were cooled back to $303 \mathrm{~K}$ and scans were repeated.

\subsection{Crystallization}

Initial crystallization trials were carried out using Crystal Screens (Hampton Research, USA). This resulted in forked crystals which grew in condition No. 22 of Crystal Screen I, the composition of which was $0.2 M$ sodium acetate trihydrate, $0.1 M$ Tris- $\mathrm{HCl} \mathrm{pH} 8.5$, $30 \%(w / v)$ PEG 4000 . The crystal quality was improved by varying the choice and concentrations of salt and PEG. Good crystals were obtained by employing the hanging-drop vapour-diffusion method, in which $1 \mu \mathrm{l}$ protein solution mixed with $1 \mu \mathrm{l}$ well solution comprising of $0.2 \mathrm{M}$ ammonium acetate, $0.1 \mathrm{M}$ Tris- $\mathrm{HCl} \mathrm{pH} 8.5,25 \%(w / v)$ PEG 2K MME was equilibrated against $1 \mathrm{ml}$ well solution. The well solution also contained $50 \mu \mathrm{l} 0.5 \%(w / v) n$-octyl $\beta$-D-glucopyranoside as an additive. All crystallization experiments were carried out at $295 \mathrm{~K}$.

\subsection{Diffraction data collection, processing and structure} determination

$\mathrm{X}$-ray diffraction data were collected on an R-AXIS $\mathrm{IV}^{++}$image plate using $\mathrm{Cu} K \alpha$ radiation generated by a Rigaku rotating-anode X-ray generator (RUH-3R) operated at $50 \mathrm{kV}$ and $100 \mathrm{~mA}$. X-rays were focused using a confocal mirror system (Osmic, USA). The crystal was kept frozen at $113 \mathrm{~K}$ in a liquid-nitrogen cryostream produced by an X-Stream system (Rigaku-MSI, USA) during data collection, using $30 \%(v / v)$ glycerol in the crystallization solution as cryoprotectant. The crystal-to-detector distance was kept at $200 \mathrm{~mm}$ and an oscillation of $0.5^{\circ}$ per frame was used. The programs DENZO and SCALEPACK (Otwinowski \& Minor, 1997) were used for processing and scaling the data. The structure was determined using the molecular-replacement method implemented in the AMoRe (Navaza, 1994) program from the CCP4 suite (Collaborative Computational Project, Number 4, 1994). Coordinates of related type II RIPs were used for modelling.

\section{Results and discussion}

\subsection{Effect of temperature}

The fluorescence emission spectra of the native lectin showed an emission maximum at $336 \mathrm{~nm}$ characteristic of $\operatorname{Trp}$ residues in a nonpolar environment. The lectin is active up to $363 \mathrm{~K}$ and the fluorescence intensity gradually decreases with increase in temperature. At $363 \mathrm{~K}$ the intensity is almost $25 \%$ of the original value. On cooling to $303 \mathrm{~K}$ the original fluorescence intensity is restored. Although Trp residues are buried, as indicated by the $\lambda_{\max }$ at $336 \mathrm{~nm}$, the microenvironments of these residues seem to be polar. No light scattering was observed under the above conditions, indicating that no aggregation of the protein molecules took place as a consequence of heating. The hydrophobic interactions in the interior of the protein molecules seem to be sufficiently strong to stabilize their respective native structures. 


\subsection{Effect of $\mathrm{Gdn}-\mathrm{HCl}$}

On treatment with increasing concentrations of $\mathrm{Gdn}-\mathrm{HCl}$, the lectin slowly started losing activity at a $3 M$ concentration of the denaturant. There was no shift in the wavelength corresponding to the emission maximum. At a $4 M$ concentration of $\mathrm{Gdn}-\mathrm{HCl} \lambda_{\max }$ shifted to $340 \mathrm{~nm}$, indicating a slight increase in the polarity of the Trp environment. A red shift in the $\lambda_{\max }$ to $355 \mathrm{~nm}$ observed at $6 \mathrm{M}$ must be a consequence of unfolding. This is accompanied by a major activity loss. At concentrations above $3 \mathrm{MGdn}-\mathrm{HCl}$, even the intensity of fluorescence has reduced substantially, indicating a change in the microenvironment of the Trp residues. Renaturation or refolding of the protein was measured by the extent of reappearance of the original spectrum and estimation of the degree of recovery of sugar-binding activity. On diluting the lectin treated with 4 and $5 \mathrm{M}$ $\mathrm{Gdn}-\mathrm{HCl}$, the activity was partially regained (with a $10-15 \%$ increase in the activity) and correspondingly a blue shift of $\lambda_{\max }$ to $340-342 \mathrm{~nm}$ was observed after $24 \mathrm{~h}$. This kind of partial renaturation and reactivation was observed also in the case of Artocarpus hirsuta lectin when 3-5 M Gdn-HCl-treated protein was diluted (Gaikwad et al., 2002).

\subsection{Effect of urea}

Just as in the case of Gdn-HCl-mediated denaturation, the lectin initially remained stable up to a $3 M$ concentration of urea and then slowly started losing activity when the urea concentration was increased to $4 M$. A major loss of activity occurred at a urea concentration of $7 \mathrm{M}$, but this was not accompanied by any major structural changes in the protein, as no significant shift in $\lambda_{\max }$ could be detected in the fluorescence. Although no major structural changes in lectin treated with urea could be observed, a comparable loss of activity as in the case of Gdn-HCl-treated lectin was observed. The reason could be that the high concentration of urea is either preventing the access of the lectin-binding site to the RBC surface or causing changes in the geometry of the binding site of the lectin itself.

\subsection{Effect of pH}

T. dioica lectin remains stable in the $\mathrm{pH}$ range 6-12 and is only partially stable at $\mathrm{pH} 4$, while $60 \%$ activity is lost at $\mathrm{pH} 2$. The fluorescence intensity is much lower at highly acidic or highly alkaline $\mathrm{pHs}$ compared with the neutral $\mathrm{pH}$ range. Thus, except for some minor changes in the microenvironment of the Trp residue, no structural transition occurrs over a wide $\mathrm{pH}$ range. The decrease in

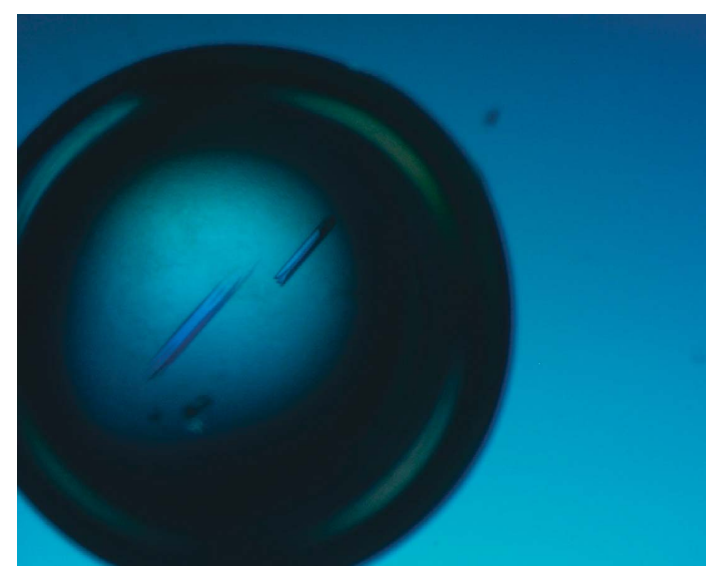

Figure 1

Hexagonal crystals of $T$. dioica lectin, maximum dimension $0.5 \mathrm{~mm}$.
Table 1

Diffraction data statistics for T. dioica lectin.

Values in parentheses are for the last shell.

\begin{tabular}{ll}
\hline Temperature $(\mathrm{K})$ & 295 \\
X-ray source & Rotating anode \\
Wavelength $(\AA)$ & $1.5418(\mathrm{Cu} K \alpha)$ \\
Resolution limits $(\AA)$ & $40.0-2.80(2.9-2.8)$ \\
No. of measured reflections & 148229 \\
No. of unique reflections & 30702 \\
Completeness of data $(\%)$ & $99.8(99.5)$ \\
$R_{\text {merge }}^{\dagger}(\%)$ & $10.9(38.4)$ \\
Average $I / \sigma(I)$ & $9.9(3.7)$ \\
Mosaicity & 0.6 \\
Space group & $P 6_{4}$ \\
Unit-cell parameters $(\AA)$ & $a=b=167.54, c=77.42$ \\
Unit-cell volume $\left(\AA^{3}\right)$ & 1882020 \\
Matthews coefficient $\left(\AA^{3} \mathrm{Da}^{-1}\right)$ & 2.85 \\
No. of molecules per unit cell $(Z)$ & 12 \\
Solvent content $(\%)$ & 56.8 \\
$R$ factor $/ R_{\text {free }}($ after first cycle) & $0.38 / 0.46$ \\
Correlation factors & $0.69 / 0.54$ \\
Reflections in working set/test set & $29807 / 1544$ \\
\hline
\end{tabular}

Reflections in working set/test set

$\dagger R_{\text {merge }}=\sum_{h k l} \sum_{i}\left|I_{i}(h k l)-\langle I(h k l)\rangle\right| / \sum_{h k l} \sum_{i} I_{i}(h k l)$.

fluorescence intensity may arise from protonated and deprotonated forms of the amino acids on the surface that affect the emission of the Trp residues.

To assess the role of disulfide linkages in the unusual stability of the lectin structure, thermal and $\mathrm{Gdn}-\mathrm{HCl}$ denaturation studies were carried out in the presence of $5 \mathrm{~m} M$ DTT as well as $5 \mathrm{~m} M$ $\beta$-mercaptoethanol. In both cases, proteins were found to be stable up to $358 \mathrm{~K}$, ruling out involvement of disulfide linkages in stabilizing the structures of Trichosanthes lectins. Previous reports and our own structural investigations indicate that both the lectins contain two non-identical subunits linked by a disulfide bond (Anuradha \& Bhide, 1999; Sultan et al., 2004).

\subsection{Crystallization and crystal characterization}

Needle-shaped crystals of $T$. dioica lectin (Fig. 1) were grown against $1 \mathrm{ml}$ well solution consisting of $0.2 \mathrm{M}$ ammonium acetate, $0.1 M$ Tris- $\mathrm{HCl} \mathrm{pH} 8.5,25 \%(w / v)$ PEG $2 \mathrm{~K}$ MME along with $50 \mu \mathrm{l}$ $0.5 \%(w / v) n$-octyl $\beta$-D-glucopyranoside as an additive. $30 \%(v / v)$ glycerol introduced into the crystallization solution acted as cryoprotectant. Crystals diffracted to $2.8 \AA$ Bragg spacing. Datacollection parameters and intensity statistics are listed in Table 1.

\subsection{Structure determination and comparison}

Initially, we could not accurately determine the space group of $T$. dioica crystals because of difficulty in fixing the screw symmetry from systematic absences of reflections. However, on running AMoRe for the translation function separately for all possible sixfold rotation axes, inputting the correct rotation-function solutions, the exact screw translation could be identified through higher correlation and lower $R$ factors of the solution (Table 2).

On carrying out the molecular-replacement procedure for structure determination, inputting the coordinates of various available type II RIPs, the coordinates of T. kirilowii lectin (PDB code 1ggp) gave the best results (Table 3), followed by those of abrin-a (PDB code 1 abr). The asymmetric unit contains two heterodimers of the $A$ and $B$ chains of the input model. The Matthews coefficient (Matthews, 1968) calculated (Table 1) assuming two heterodimers in the asymmetric unit was also within the expected range. Removing the first residues $1-8$ of the $B$ chain and residues $41-49$ of the $A$ chain in abrin-a gave slightly improved correlation parameters compared with inputting the whole abrin-a molecule. In fact, in the solution with 
Table 2

Calculation of translation function to obtain the correct space group.

The solutions with the correct space group input have a comparatively higher correlation factor and lower $R$ factor and are highlighted in bold.

\begin{tabular}{|c|c|c|c|}
\hline Input space group & Rotation-function solution & Correlation factor & $R$ factor $(\%)$ \\
\hline \multirow[t]{2}{*}{$P 6$} & 1 & 30.0 & 54.7 \\
\hline & 2 & 29.8 & 54.7 \\
\hline \multirow{2}{*}{$P 6_{1}$} & 1 & 32.9 & 53.5 \\
\hline & 2 & 31.2 & 54.1 \\
\hline \multirow[t]{2}{*}{$P 6_{2}$} & 1 & 30.0 & 54.5 \\
\hline & 2 & 29.6 & 54.7 \\
\hline \multirow[t]{2}{*}{$\mathrm{PG}_{3}$} & 1 & 30.3 & 54.4 \\
\hline & 2 & 29.5 & 54.8 \\
\hline \multirow[t]{2}{*}{$\mathrm{P6}_{4}$} & 1 & 37.2 & 52.1 \\
\hline & 2 & 34.5 & 53.2 \\
\hline \multirow[t]{2}{*}{$P 6_{5}$} & 1 & 30.2 & 54.5 \\
\hline & 2 & 29.5 & 54.6 \\
\hline
\end{tabular}

Table 3

The molecular-replacement solutions obtained using the T. kirilowii lectin structure as model.

The parameters shown are after rigid-body refinement in $A M o R e$ using reflections in the resolution range $20-2.8 \mathrm{~A}$. (CC, correlation coefficient; $R, R$ factor.)

\begin{tabular}{llrllllll}
\hline Solution & $\alpha\left({ }^{\circ}\right)$ & $\beta\left({ }^{\circ}\right)$ & $\gamma\left({ }^{\circ}\right)$ & $T_{x}$ & $T_{y}$ & $T_{z}$ & $\mathrm{CC}$ & $R$ \\
\hline 1 & 45.30 & 87.44 & 146.85 & 0.7491 & 0.5007 & -0.0002 & 48.6 & 48.5 \\
2 & 22.03 & 135.53 & 317.97 & 0.7153 & 0.7702 & -0.0007 & & \\
\hline
\end{tabular}

the whole abrin-a molecule the deleted residues made short contacts with the second molecule of the asymmetric unit. The report of the structure determination of $T$. anguina lectin also mentions the removal of similar residues from the input model (Manoj et al., 2001). The parameters for the second solution were far below those of the first correct solution when ricin coordinates (PDB code 2aai) were used as an input model in the rotation function. Inputting corresponding polyalanine models into $A M o R e$ only worsened the solution parameters. Graphic visualization of the crystal packing of correct solutions selected showed no serious short contacts between neighbouring molecules.

In the absence of sequence information, no extensive refinement could be attempted on the initial model. However, an estimated $R$ factor of 0.38 and $R_{\text {free }}$ of 0.46 were obtained on applying one cycle of refinement (subsequent to rigid-body refinement) prior to electrondensity calculation using REFMAC (Murshudov et al., 1997), tightly holding the non-crystallographic restraint between the two molecules in the asymmetric unit. The corresponding correlation factors after one cycle of refinement were 0.69 and 0.54 for reflections in the resolution range $25-2.8 \AA$. By overlapping the initial model on the electron-density map, it could be confirmed that the gross features of the type II RIP structural elements were also conserved in the structure of the $T$. dioica lectin. Out of the five residues, Tyr74, Tyr113, Glu164, Arg167 and Trp198, of the $A$ chain of abrin-a identified as important for the ribosome-inactivating mechanism, only the latter four are conserved in the $T$. dioica lectin. There is no sufficient electron density observed in the preliminary electron-density map to accommodate the aromatic side chain of tyrosine for residue 74 . This feature makes this lectin closer to both the T. anguina and T. kirilowii lectins, which also reportedly only have electron density for an Ala residue at this position. All the disulfide bonds found in T. kirilowii lectin, including the inter-chain disulfide bond between the $A$ and $B$ chains, seem to be preserved in this lectin.

One major difference between the present crystal structure and those of the T. anguina and T. kirilowii lectins is the association of the two heterodimers in the asymmetric unit and the crystal packing. The association of the two molecules of the $T$. dioica lectin in the asymmetric unit takes place exclusively through interactions involving the $A$ chains (Fig. 2). This type of dimer formation is not observed in the crystals of other two Trichosanthes lectins.

Unfortunately, in the absence of confirmed amino-acid sequence for any of the three Trichosanthes lectins, any detailed attempts to correlate stability with structural features at this time may be premature and inconclusive. However, the study reported is highly suggestive of a close relationship between the structural and biochemical characteristics and the type II RIP features common to the three Trichosanthes lectins of $T$. dioica, T. anguina and T. kirilowii.

\section{Conclusions}

Type II ribosome-inactivating proteins (type II RIPs) are toxic $\mathrm{N}$-glycosidases that depurinate the universally conserved sarcin loop of larger rRNAs. RIPs are widely distributed among different plant genera and are present in a variety of different tissues. Some of them are highly thermostable ( $\mathrm{Lam} \& \mathrm{Ng}$, 2001). It has been pointed out that Trichosanthes lectins have a similarity to type II RIPs (Sultan et al., 2004); here, we have shown that like other type II RIPs, the T. dioica lectin is also resistant to a wide range of denaturing conditions. We have further substantiated this observation by determining its structure and proving that structurally also it belongs to the class of type II RIPs. However, our data shows that stability is not directly influenced by the presence of structural elements such as disulfide bridges alone. Furthermore, even in the absence of any confirmed amino-acid sequences, we have demonstrated through correlation of gross three-dimensional structural features that the

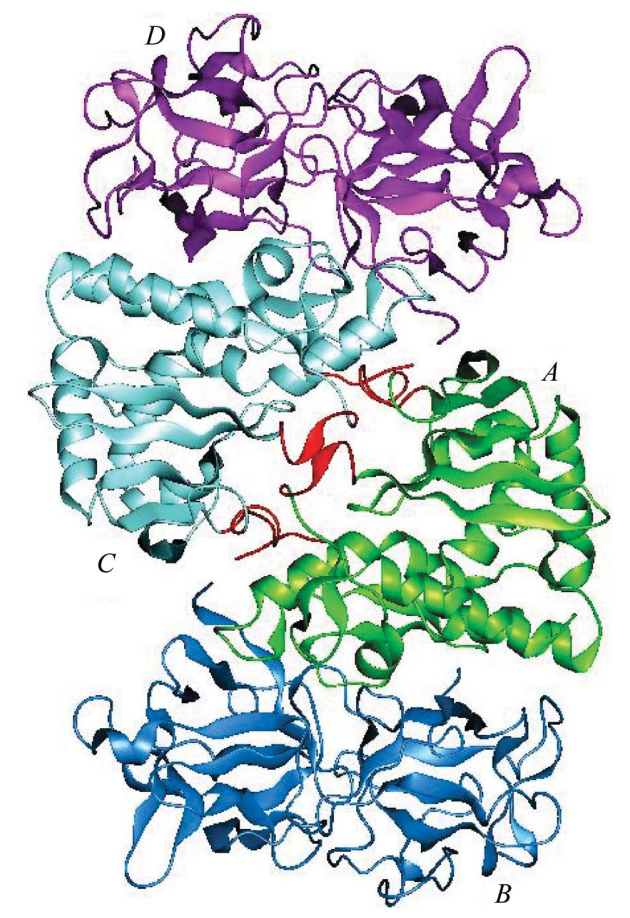

Figure 2

The dimer-dimer association of the two heterodimer molecules of $T$. dioica lectin in its crystal structure. The segments are coloured differently and the subunits are labelled $A, B, C$ and $D$. The two $A$ chains (subunits $A$ and $C$ ) come closer in the asymmetric dimer. The parts of the molecules in the interface are shown in red. Strands from the $A$ and $C$ subunits (residues 45-47 with respect to T. kirilowii lectin) form a short $\beta$-sheet in the middle of the interface. 
three Trichosanthes lectins from T. dioica, T. anguina and T. kirilowii are evolutionarily closely related.

PDD is Senior Research Fellow of the Council of Scientific and Industrial Research, New Delhi. PA acknowledges the Department of Biotechnology, New Delhi, India for a Post-Doctoral Fellowship.

\section{References}

Ahmed, N., Srinivas, V. R., Reddy, G. B. \& Surolia, A. (1998). Biochemistry, 37, 16765-16772.

Anuradha, P. \& Bhide, S. V. (1999). Phytochemistry, 52, 751-758.

Collaborative Computational Project, Number 4 (1994). Acta Cryst. D50, 760-763.

Gaikwad, S. M., Gurjar, M. M. \& Khan, M. I. (2002). Eur. J. Biochem. 269, 1413-1417.

Gaikwad, S. M. \& Khan, M. I. (2003). Biochem. Biophys. Res. Commun. 311, 254-257.

Hartley, M. R. \& Lord, J. M. (2004). Biochim. Biophys. Acta, 1701, 1-14.

Komath, S. S., Nadimpalli, S. K. \& Swamy, M. J. (1998). Biochem. Mol. Biol. Int. 44, 107-116.

Komath, S. S. \& Swamy, M. J. (1999). J. Photochem. Photobiol. B, Biol. 50, 108-118.

Krauspenhaar, R., Eschenburg, S., Perbandt, M., Kornilov, V., Konareva, N., Milkailova, I., Stoeva, S., Wacker, R., Maier, T., Singh, T., Mikhailov, A.,
Voelter, W. \& Betzel, C. (1999). Biochem. Biophys. Res. Commun. 257, 418-424.

Lam, S. K. \& Ng, T. B. (2001). Biophys. Biochem. Res. Commun. 285, 10711075.

Li, M., Chai, J. J., Wang, Y. P., Wang, K. Y. \& Bi, R.-C. (2001). Protein Pept. Lett. 8, 81-87.

Lis, H. \& Sharon, N. (1991). Curr. Opin. Struct. Biol. 246, 227-234.

Manoj, N., Jeyaprakash, A. A., Pratap, J. V., Komath, S. S., Kenoth, R., Swamy, M. J. \& Vijayan, M. (2001). Acta Cryst. D57, 912-914.

Matthews, B. W. (1968). J. Mol. Biol. 33, 491-497.

Montfort, W., Villafranca, J. E., Monzingo, A. F., Ernst, S. R., Katzin, B., Rutenber, E., Xuong, N.-H., Hamlin, R. \& Roberts, J. D. (1987). J. Biol. Chem. 262, 5398-5403.

Murshudov, G. N., Vagin, A. A. \& Dodson, E. J. (1997). Acta Cryst. D53, 240-255.

Navaza, J. (1994). Acta Cryst. A50, 157-163.

Otwinowski, Z. \& Minor, W. (1997). Methods Enzymol. 276, 307-326.

Reddy, G. B., Srinivas, V. R., Ahmed, N. \& Surolia, A. (1999). J. Biol. Chem. 274, 4500-4503.

Sahasrabuddhe, A. A., Gaikwad, S. M., Krishnasastry, M. V. \& Khan, M. I. (2004). Protein Sci. 13, 3264-3273.

Sultan, N. A. M., Kenoth, R. \& Swamy, M. J. (2004). Arch. Biochem. Biophys. 432, 212-221.

Sultan, N. A. M. \& Swamy, M. J. (2005). J. Photochem. Photobiol. B, Biol. 80, 93-100.

Tahirov, T. H., Lu, T.-H., Liaw, Y.-C., Chen, Y.-L. \& Lin, J.-Y. (1995). J. Mol. Biol. 250, 354-367. 